Check for updates

Cite this: Soft Matter, 2020, 16,5565

Received 20th March 2020, Accepted 1st May 2020

DOI: $10.1039 / \mathrm{d} 0 \mathrm{sm} 00484 \mathrm{~g}$

rsc.li/soft-matter-journal

\section{Self-assembly of freely-rotating polydisperse cuboids: unveiling the boundaries of the biaxial nematic phase $\uparrow$}

\author{
Effran Mirzad Rafael, (DD ${ }^{a}$ Daniel Corbett, ${ }^{a}$ Alejandro Cuetos $\left(D^{b}\right.$ and \\ Alessandro Patti (iD *a
}

Colloidal cuboids have the potential to self-assemble into biaxial liquid crystal phases, which exhibit two independent optical axes. Over the last few decades, several theoretical works have predicted the existence of a wide region of the phase diagram where the biaxial nematic phase would be stable, but imposed rather strong constraints on the particle rotational degrees of freedom. In this work, we use molecular simulation to investigate the impact of size dispersity on the phase behaviour of freely-rotating hard cuboids, here modelled as self-dual-shaped nanoboards. This peculiar anisotropy, exactly in between the oblate and prolate geometry, has been proposed as the most appropriate to promote phase biaxiality. We observe that size dispersity radically changes the phase behaviour of monodisperse systems and leads to the formation of an elusive biaxial nematic phase, being found in a large region of the packing fraction vs. polydispersity phase diagram. Although our results confirm the tendencies reported in past experimental observations on colloidal dispersions of slightly prolate goethite particles, they cannot reproduce the direct isotropic-to-biaxial nematic phase transition observed in these experiments.

The first known theory on entropy-driven phase transitions was proposed by Onsager in his seminal work dating back to the 1940s. ${ }^{1}$ Onsager demonstrated that systems of infinitely long hard rods exhibit an isotropic-to-nematic phase transition as a result of mere volume effects. Later on, experiments and computer simulations showed that entropy-driven phase transitions can also lead to the formation of positionally ordered liquid crystals (LCs), such as smectic and columnar phases. ${ }^{2,3}$ The equilibrium structures stemming from the self-assembly of colloidal particles are especially determined by the architecture of their building

\footnotetext{
${ }^{a}$ Department of Chemical Engineering and Analytical Science,

The University of Manchester, Manchester, M13 9PL, UK.

E-mail:alessandro.patti@manchester.ac.uk

${ }^{b}$ Department of Physical, Chemical and Natural Systems, Pablo de Olavide

University, 41013 Sevilla, Spain

$\dagger$ Electronic supplementary information (ESI) available: Details of order parameters, pair distribution functions, smectic layer thicknesses, free energies and equation of state. See DOI: $10.1039 / \mathrm{d} 0 \mathrm{sm} 00484 \mathrm{~g}$
}

blocks. In particular, biaxial particles, such as bent-core and cuboidal particles, have been reported to form biaxial nematic $\left(\mathrm{N}_{\mathrm{B}}\right)$ LCs. $^{4-6}$ First theorised by Freiser in $1970,{ }^{7}$ the $N_{B}$ phase has attracted widespread attention for being a promising candidate to be engineered into next generation liquid crystal displays. In contrast to uniaxial nematics $\left(\mathrm{N}_{\mathrm{U}}\right)$, where long-range orientational order exists only along one direction, the $\mathrm{N}_{\mathrm{B}}$ phase possesses three orthogonal directors and hence two distinct optical axes that can pave the path to high-performance displays. ${ }^{8-10}$ Despite having been extensively studied over the past 50 years, the stability of the $\mathrm{N}_{\mathrm{B}}$ phase still remains an open question, predominantly, but not only, at the molecular scale. Answering this question is hampered by the fact that the $\mathrm{N}_{\mathrm{B}}$ phase tends to be metastable with respect to other morphologies, such as the $\mathrm{N}_{\mathrm{U}}$ and Sm phases. ${ }^{11}$

About a decade ago, Vroege and coworkers reported the first experimental evidence of the $\mathrm{N}_{\mathrm{B}}$ phase in a system of colloidal goethite (roughly board-like) particles. ${ }^{6}$ The stability of this $\mathrm{N}_{\mathrm{B}}$ phase was ascribed to the particles' quasi self-dual shape, a geometry in between oblate and prolate, and to their significant size dispersity, which hinders the formation of the Sm phase. This key work has reignited recent interest, sparking numerous theoretical, experimental and computer simulation studies on hard board-like particles (HBPs) ${ }^{12-27}$ and other biaxial geometries. ${ }^{24,28-32}$ Theoretical and computational studies on monodisperse systems have suggested that self-dual-shaped particles exhibit a higher tendency to form biaxial nematics. However, these studies applied rather strong approximations, limiting the particle orientation to six orthogonal directions, ${ }^{11-13}$ freezing the rotation of the long axes of the particle, ${ }^{33}$ or neglecting the occurrence of positionally ordered LC phases. ${ }^{34-37}$ Our recent theoretical work and computer simulations of freely-rotating HBPs suggested that these approximations might artificially magnify the stability of the $\mathrm{N}_{\mathrm{B}}$ phase..$^{22,23}$ We note that stable $\mathrm{N}_{\mathrm{B}}$ phases have been found in systems of cuboids with rounded corners (spheroplatelets) with a length-to-thickness ratio of $L^{*} \equiv L / T>9^{16,17}$ and $L^{*} \geq 23$ in systems of especially elongated HBPs. $^{24}$ Sedimentation experiments on highly uniform and monodisperse colloidal cuboids with $15 \leq L^{*} \leq 180$ did not 
report the formation of an $\mathrm{N}_{\mathrm{B}}$ phase, which, for self-dual-shaped cuboids, was found to be pre-empted by biaxial smectic $\left(\mathrm{Sm}_{\mathrm{B}}\right)$ or crystal-B phases, depending on the solvent used. ${ }^{25}$ We stress that these conditions, probing stacking behaviour, are very different from those of ref. 24, studying bulk behaviour, and thus their conclusions are not expected to necessarily match. This lack of agreement between experiments, theories and simulations keeps the discussion on the ability of HBPs to form the $\mathrm{N}_{\mathrm{B}}$ phase alive.

Size dispersity has been identified as a key ingredient to destabilise the Sm phase and thus promote the formation of biaxial nematics. In particular, the effect of size dispersity in systems of HBPs was investigated by Onsager's theory within the restricted-orientation (Zwanzig) approximation. ${ }^{13}$ This theory provided an elegant and solid explanation on the origin of the $\mathrm{N}_{B}$ stability experimentally observed in colloidal dispersions of goethite particles. ${ }^{6}$ Nevertheless, its conclusions were strongly determined by the use of the Zwanzig model, which only allows six orthogonal particle orientations and cannot describe the phase behaviour of cuboids accurately, as recent simulations and theory have indicated. ${ }^{22,23}$ Consequently, fully unlocking the particle rotational degrees of freedom is of paramount importance to ascertain the impact of polydispersity on the phase behaviour of HBPs and accurately map the boundaries of the $\mathrm{N}_{\mathrm{B}}$ phase. While it is extremely challenging to formulate a theory that simultaneously incorporates particle size dispersity and unrestricted orientations, molecular simulation can provide an insightful contribution to shed light on this combined effect. To this end, we have performed Monte Carlo (MC) simulations of freely-rotating HBPs with Gaussian size distribution peaked at $L^{*}=12$. The particle thickness, $T$, is the system unit length and is the same for all HBPs, whereas $L^{*}$ changes with standard deviation $\sigma_{\mathrm{L}}\left\langle L^{*}\right\rangle$, where $0.05 \leq \sigma_{\mathrm{L}} \leq 0.30$ measures the particle length dispersity. Finally, the particle width dispersity is linked to the length dispersity by $W^{*} \equiv W / T=\sqrt{L^{*}}$, which ensures the self-dual shape for all particles. Our systems consist of $N_{\mathrm{p}}=2000$ to $3000 \mathrm{HBPs}$ that are initially arranged in cubic or rectangular boxes with periodic boundaries and are equilibrated in the isothermal-isobaric ensemble. Phase transitions have been assessed by expansion or compression of a perfect biaxial nematic phase at extensive range of pressures. To ensure that the equilibrium configurations were independent of the initial configuration, expansion of $\mathrm{Sm}$ phases and compression of isotropic (I) phases have also been carried out. We have also simulated significantly larger systems, with $N_{\mathrm{p}}=6000$, to discard the occurrence of finite-size effects, where these could especially influence the symmetry of the phases observed, that is at the I-N transition. Additionally, the potential occurrence of fractionation has been assessed by evaluating the space distribution of particles whose size is one standard deviation above or below the average size distribution and the space dependence of the order parameters. No evidence of fractionation, including $\mathrm{N}_{\mathrm{U}}^{+} / \mathrm{N}_{\mathrm{U}}^{-}$separation in the $\mathrm{N}_{\mathrm{B}}$ region, has been detected. Other more sophisticated techniques, such as isobaric semigrand MC simulations, ${ }^{38}$ are perhaps better suited to assess the occurrence of fractionation in polydisperse systems. Generally, up to $10^{7} \mathrm{MC}$ cycles were needed to equilibrate the systems, with a cycle consisting of $N_{\mathrm{p}}$ attempts of displacing and/or rotating randomly selected particles and one trial volume change. Because the force field used here consists only of a hard-core potential, these moves were accepted if no overlaps were detected, according to the separating axes theorem. ${ }^{39,40}$ Systems were considered to be at equilibrium if packing fraction $(\eta)$ and uniaxial $\left(S_{2}\right)$ and biaxial $\left(B_{2}\right)$ order parameters achieved steady values within reasonable statistical uncertainty. In particular, $\eta \equiv \sum_{i=1}^{N_{\mathrm{p}}} v_{i} / V$, where $V$ is the box volume and $v_{i}$ is the volume of a generic particle $i$. The calculation of $S_{2}$ and $B_{2}$ was done by diagonalisation of the traceless second-rank symmetric tensor $\mathbf{Q}^{\lambda \lambda}=\left\langle\sum_{i=1}^{N_{\mathrm{p}}}\left(3 \hat{\lambda}_{i} \cdot \hat{\lambda}_{i}-\mathbf{I}\right)\right\rangle / 2 N_{\mathrm{p}}$, where I is the second-rank unit tensor, $\hat{\lambda}_{i}=\hat{x}, \hat{y}$ and $\hat{z}$ is the particle unit orientation vector and angular brackets denotes ensemble average. Isotropic, perfect uniaxial and perfect biaxial phases are observed at $\left(S_{2}, B_{2}\right)=(0,0),(1,0)$ and $(1,1)$, respectively. An illustrative example of the dependence of the order parameters on the packing fraction is given in Fig. 1, for different values of $\sigma_{\mathrm{L}}$. Finally, to assess the long-range ordering of the phases at equilibrium, we have analysed the spatial correlations along and perpendicularly to the relevant phase directors by computing the longitudinal, $g_{\|}\left(r_{\|}\right)$, and transverse, $g_{\perp}\left(r_{\perp}\right)$, pair distribution functions, where $r_{\|}$and $r_{\perp}$ are the corresponding projections of the inter-particle distance. Interested readers can refer to the ESI $\dagger$ for further details on the calculation of order parameters and pair correlation functions.

Bearing in mind these introductory considerations, we now report on the LC phases that polydisperse HBPs are able to form at the equilibrium, with specific interest in the critical polydispersity that stabilizes the $\mathrm{N}_{\mathrm{B}}$ phase and the extent of this stability. For consistency with our former work, ${ }^{26}$ we defined the axial symmetry of the $\mathrm{N}_{\mathrm{B}}$ and Sm phases according to the magnitude of the order parameters (see the ESI $\dagger$ for additional details). The $\sigma_{\mathrm{L}}-\eta$ phase diagram of polydisperse and

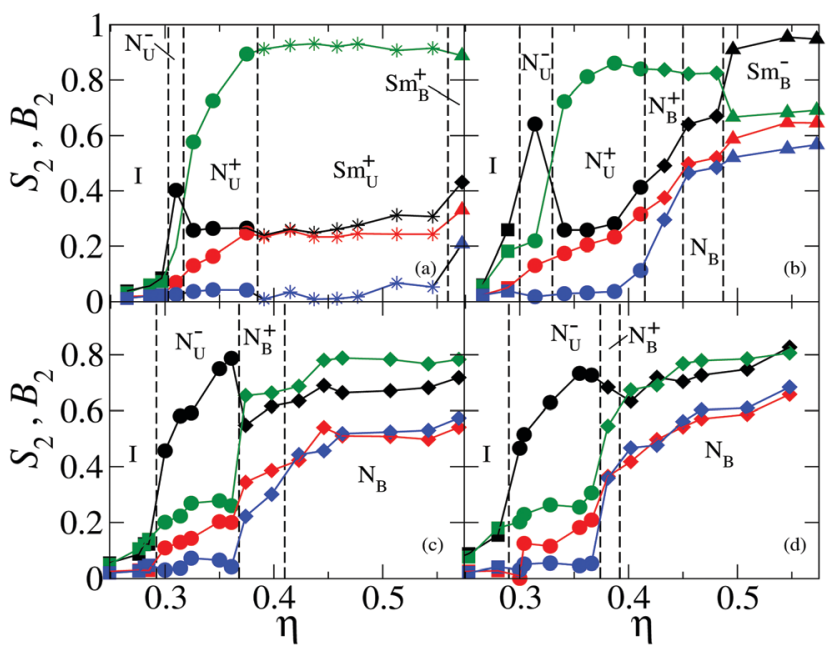

Fig. 1 Uniaxial and biaxial order parameters as a function of the packing fraction at $\sigma_{\mathrm{L}}=0.05$ (a), 0.18 (b), 0.25 (c), and 0.30 (d). Black, red, green, and blue symbols refer to $S_{2, T}, S_{2, W}, S_{2, L}$ and $B_{2}$, respectively. 


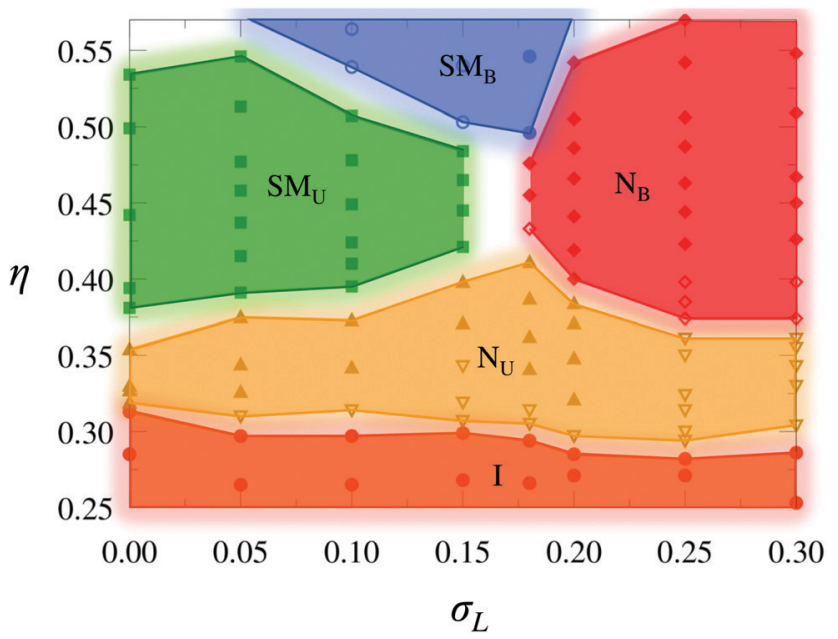

Fig. 2 Phase diagram of polydisperse and freely-rotating HBPs with $\left\langle L^{*}\right\rangle=12,\left\langle W^{*}\right\rangle=\sqrt{12} \approx 3.46$ and length polydispersity $0 \leq \sigma_{\mathrm{L}} \leq 0.30$ The following phases are identified: I $(\diamond), N_{\cup}^{-}(\nabla), N_{\cup}^{+}(\Delta), N_{B}^{+}(\diamond), N_{B}(\diamond)$, $\mathrm{Sm}_{\cup}^{+}(\square), \mathrm{Sm}_{\mathrm{B}}^{+}(\bigcirc)$, and $\mathrm{Sm}_{\mathrm{B}}^{-}(\bigcirc)$.

self-dual-shaped HBPs is shown in Fig. 2. All points at $\sigma_{\mathrm{L}}=0$ refer to monodisperse systems, obtained from previous simulation results ${ }^{22}$ and included here as a reference. The diagram reveals the existence of uniaxial and biaxial LC phases at $\eta \gtrsim 0.30$. At a lower density and regardless of the size dispersity, we only detect I phases, in good agreement with the Zwanzigbased Onsager theory by Belli et al., who predicted stable I phases for $\eta \lesssim 0.28$ and $0 \leq \sigma_{\mathrm{L}} \leq 0.4 .^{13}$ We stress that these authors studied slightly prolate particles with $W^{*} \approx 1.017 \sqrt{L^{*}}$ or $\nu \equiv L / W-W / T=0.1$. This slight deviation from the self-dual shape, for which $\nu=0$, might appear insignificant, but in fact it determines the oblate or prolate symmetry of the nematic phase, at least in monodisperse systems, as established by Mulder in the 1980 s. $^{35}$
As far as the $\mathrm{N}_{\mathrm{U}}$ phase is concerned, its stability region (orange-shaded area in Fig. 2) is not especially influenced by the polydispersity of the particle, with the lower and upper boundaries approximately constrained between $\eta=0.30$ and 0.35 , similar to those observed in monodisperse systems $\left(\sigma_{\mathrm{L}}=0\right)$. A slight difference is detected at $0.15 \leq \sigma_{\mathrm{L}} \leq 0.20$, where four different phases seem to converge and the region of stability of uniaxial nematics expands up to $\eta=0.41$ at $\sigma_{\mathrm{L}}=0.18$. In former simulation studies on monodisperse systems of self-dual-shaped HBPs with $L^{*}=12$, the $N_{U}$ stability was also observed to be relatively small, ${ }^{24}$ and even to vanish in binary mixtures. ${ }^{23}$ Within the $\mathrm{N}_{\mathrm{U}}$ domain, we came across nematic LCs with oblate and prolate symmetries, respectively labelled as $\mathrm{N}_{\mathrm{U}}^{-}$and $\mathrm{N}_{\mathrm{U}}^{+}$. The occurrence of the $\mathrm{N}_{\mathrm{U}}^{-}$phase is predominantly detected close to the I-N transition and also far from it for $\sigma_{\mathrm{L}} \geq 0.25$. In contrast, the $\mathrm{N}_{\mathrm{U}}^{+}$phase is mostly observed at larger packing fractions for $0 \leq \sigma_{\mathrm{L}} \leq 0.20$. Consequently, at a given polydispersity, increasing the system density can produce an inversion of the nematic phase symmetry. Meanwhile, the prolate and oblate particles commonly tend to trigger the $\mathrm{N}_{\mathrm{U}}^{+}$and $\mathrm{N}_{\mathrm{U}}^{-}$phases, respectively. ${ }^{11,18,34,35,37,41}$ Although a significant polydispersity can counteract this tendency ${ }^{13}$ the self-dual shape is, in principle, not expected to exhibit a clear oblate or prolate nature, but rather an ambivalent nature. By applying our Onsager-like theory, ${ }^{22}$ we observed that, close to the I-N transition, the free-energy difference between $\mathrm{N}_{\mathrm{U}}^{+}$ and $\mathrm{N}_{\mathrm{U}}^{-}$phases is very small and not much larger at increasing $\eta$ (see ESI†). Although this theory is strictly valid for monodisperse systems and serves here as a mere qualitative guideline, it confirms the very small free-energy differences between oblate and prolate symmetries that had also been reported by Martnez-Ratón and co-workers, who applied density-functional theory to study the phase behaviour of nearly self-dual-shaped monodisperse HBPs. ${ }^{12}$

Uniaxial and biaxial smectic phases are obtained at larger packing fractions. To distinguish them from the nematic phases, we calculated the density distribution function along

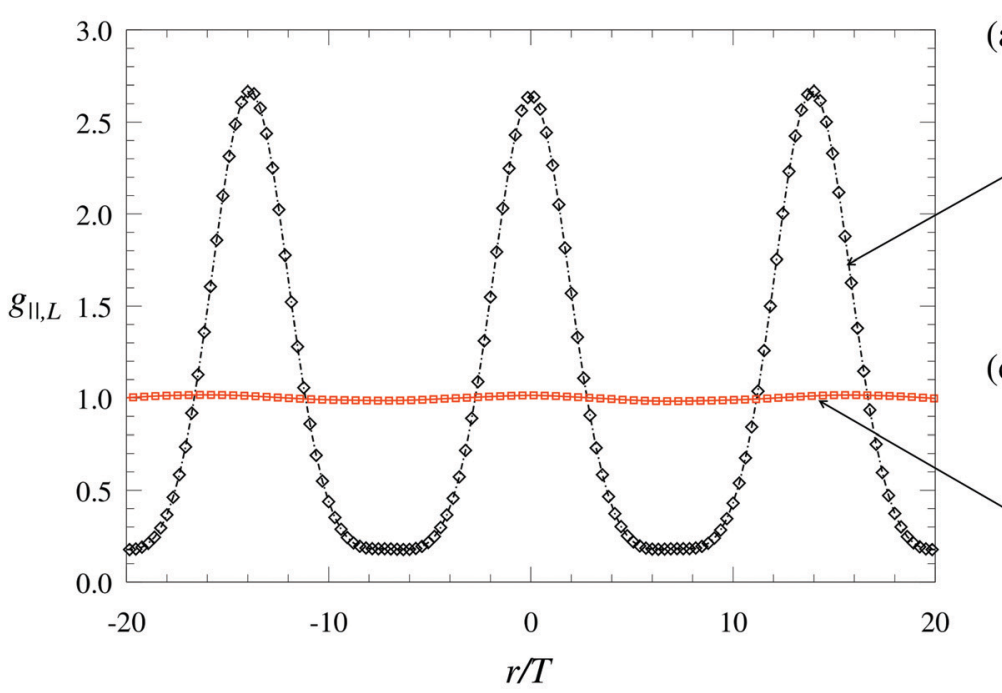

(a)

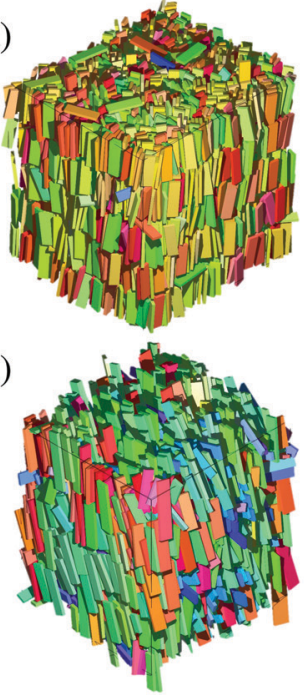

(b)

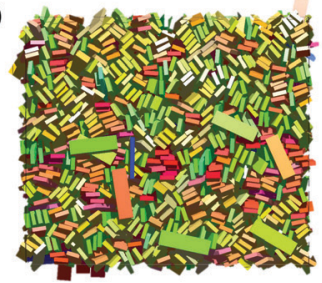

(d)

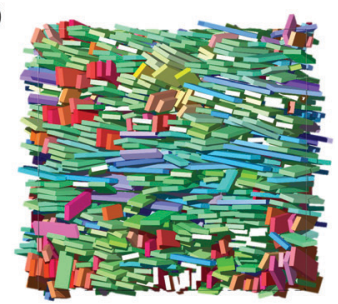

Fig. 3 Parallel pair distribution functions along the nematic director of ((a) and (b)) a uniaxial smectic phase of prolate symmetry at $\sigma_{\mathrm{L}}=0.05$ and $\eta=0.415(\diamond)$, and ((c) and (d)) a biaxial nematic phase at $\sigma_{\mathrm{L}}=0.25$ and $\eta=0.423(\square)$. Different colours indicate different particle orientations. 
the nematic director, $g_{\|}\left(r_{\|}\right)$, identified by the order parameters. Uniaxial smectic $\left(\mathrm{Sm}_{\mathrm{U}}\right)$ phases (green area in Fig. 2 ) only exhibit a prolate symmetry $\left(\mathrm{Sm}_{\mathrm{U}}^{+}\right)$, in agreement with former simulations of monodisperse systems. ${ }^{22}$ In particular, their layers, whose thickness is roughly $13 T$ to $15 T$, are perpendicular to the average direction of the particle length. The $g_{\|}\left(r_{\|}\right)$, calculated along this direction, displays periodically peaked profiles of the type reported in Fig. 3 and displays no indication of structural order in the other directions. In contrast, biaxial smectics, found at $\eta>0.50$ and $0.05 \leq \sigma_{\mathrm{L}} \leq 0.20$ (blue area in Fig. 2), can present a prolate $\left(\mathrm{Sm}_{\mathrm{B}}^{+}\right)$or oblate $\left(\mathrm{Sm}_{\mathrm{B}}^{-}\right)$symmetry. The former is characterised by layers piling along the particle length, and the latter by layers piling along the particle thickness. Upon increasing polydispersity, the $\mathrm{Sm}_{\mathrm{B}}$ phase acquires a more and more defined structural identity, with a weak-to-strong biaxiality crossover at approximately $0.05<\sigma_{\mathrm{L}}<0.10$ and a full biaxial character $\left(B_{2}>0.6\right)$ at $\sigma_{\mathrm{L}} \geq 0.18$. At this value of size dispersity, our simulations highlighted a particularly rich phase behaviour, unveiling an $\mathrm{I} \rightarrow \mathrm{N}_{\mathrm{U}} \rightarrow \mathrm{N}_{\mathrm{B}} \rightarrow \mathrm{Sm}_{\mathrm{B}}$ sequence of phases that is exemplarily shown in Fig. 4. In qualitative agreement with theory and experiments, we found the $\mathrm{N}_{\mathrm{B}}$ phase to be stabilised by a substantial degree of particle size dispersity. In particular, our simulation results indicate $\sigma_{\mathrm{L}}=0.18$ as the critical polydispersity above which the $\mathrm{N}_{B}$ phase can form. Close to the $\mathrm{N}_{\mathrm{U}}-\mathrm{N}_{\mathrm{B}}$ phase boundary, we find nematics with a relatively weak (but non-negligible) biaxiality and a residual oblate or prolate character. These phases, here referred to as weak biaxial nematics, are characterised by a biaxial order parameter in the range $0.2 \leq B_{2} \leq 0.30$ and one predominant uniaxial order parameter granting them prolate $\left(\mathrm{N}_{\mathrm{B}}^{+}\right)$or oblate $\left(\mathrm{N}_{\mathrm{B}}^{-}\right)$symmetry.

In contrast with the experiments on goethite particles, ${ }^{6}$ we do not observe a direct $\mathrm{I}-\mathrm{N}_{\mathrm{B}}$ phase transition here. This apparent lack of agreement deserves some comments. First of all, the cuboids studied in these experiments are not self-dual-shaped. Their shape parameter, $\nu=0.1$, indicates a prolate geometry, which in monodisperse systems is expected to promote an $\mathrm{I}-\mathrm{N}_{\mathrm{U}}^{+}$, rather than $\mathrm{I}-\mathrm{N}_{\mathrm{B}}$, transition as predicted by theories spanning almost five decades. ${ }^{11,18,34,35,37,41}$ However, the goethite particles utilized by Vroege and co-workers are not monodisperse, but exhibit a polydispersity between $20 \%$ and $25 \%$ in the three directions. Because polydispersity can lead to fractionation ${ }^{42}$ and these authors studied the phase behaviour in capillaries, the longer particles tend to accumulate towards the bottom, where the $\mathrm{N}_{\mathrm{B}}$ phase was found, de facto increasing the shape parameter of this subset of particles to the effective value of $\nu=0.6 .^{6}$ This particle geometry, evidently prolate, is very different from the selfdual shape applied here and a quantitative analogy is therefore not directly possible. The Onsager theory within the Zwanzig approximation does not predict a direct $\mathrm{I}-\mathrm{N}_{\mathrm{B}}$ transition in systems of polydisperse HBPs with $\nu=0.1$, but it suggests the existence of the $\mathrm{N}_{\mathrm{B}}$ phase in a wide region of the $\eta-\sigma_{\mathrm{L}}$ phase diagram, including for $\sigma_{\mathrm{L}}<0.1 .^{13}$ While it is known that restricting orientations can significantly enhance the stability of the $\mathrm{N}_{\mathrm{B}}$ phase, both Belli's theoretical work and our simulations do not report a direct $\mathrm{I}-\mathrm{N}_{\mathrm{B}}$ phase transition, whose existence has never been unambiguously confirmed by off-lattice simulations spanning more than twenty years. ${ }^{16,24,30,37,43-46}$ Indeed, our recent MC simulations and generalised fifth-virial Onsager theory applied to freely-rotating monodisperse HBPs with $L^{*} \leq 12$ had even excluded the existence of the $\mathrm{N}_{B}$ phase, also at the self-dual shape. A third-virial theory for monodisperse cuboids with continuum orientations predicted a direct $\mathrm{I}-\mathrm{N}_{\mathrm{B}}$ transition at $L^{*}=64$, which was not observed in the simulations by the same authors. ${ }^{24}$ To the best of our knowledge, there are no theoretical works on freely-rotating polydisperse HBPs that might help resolve this conundrum. While the phase diagram in Fig. 2 illustrates the relevant discrepancies of the work proposed by Belli, both works agree very well on the key role of polydispersity in the stabilisation of the $\mathrm{N}_{\mathrm{B}}$ phase. This is especially evident at $\sigma_{\mathrm{L}} \geq 0.18$, where the stability region of the $\mathrm{N}_{\mathrm{B}}$ phase widens, remarkably reducing that of the $\mathrm{Sm}$ and $\mathrm{N}_{\mathrm{U}}$ phases. This is not surprising as a large size dispersity is expected to hinder the formation of layered structures due to the absence of welldefined structural periodicity in the longitudinal direction.

In summary, our MC simulations of freely-rotating HBPs have revealed a rich phase behaviour that is characterised by three key results: (i) a significant degree of particle size dispersity is needed to stabilise the $\mathrm{N}_{B}$ phase; (ii) self-dual-shaped HBPs do not exhibit a direct $\mathrm{I}-\mathrm{N}_{B}$ phase transition in the range of size dispersities studied here; (iii) the ambivalent nature of the self-dual shape provides uniaxial nematics that, in a relatively wide region of the $\eta-\sigma_{\mathrm{L}}$ phase diagram, might well be oblate or prolate.
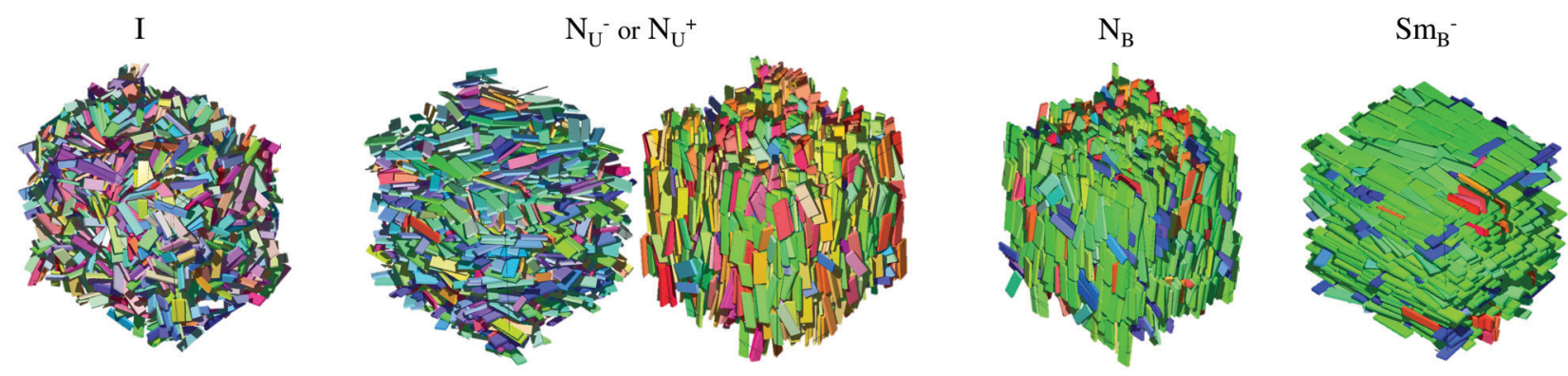

Packing fraction, $\eta$

Fig. 4 Equilibrium phases of HBPs at $\sigma_{\mathrm{L}}=0.18 \mathrm{in}$ an isotropic phase $(\eta=0.266)$, oblate nematic phase $(\eta=0.314)$, prolate nematic phase $(\eta=0.341)$, biaxial nematic phase $(\eta=0.455)$ and a biaxial smectic phase of oblate symmetry $(\eta=0.573)$. Different colours indicate different particle orientations. 
More specifically, a significant polydispersity $\left(\sigma_{\mathrm{L}} \geq 0.18\right)$ prevents the discretization of the space along the nematic director and thus enfeebles the stability of the Sm phase, practically enhancing that of the nematic phase. This result is in line with the experiments and theories, but on a merely qualitative basis only. The lack of a direct I- $\mathrm{N}_{\mathrm{B}}$ phase transition might appear in evident disagreement with previous experimental observations, which anyway used prolate rather than self-dual-shaped particles, but agrees with a relevant number of simulation studies that could not confirm its occurrence in a reasonable range of particle anisotropies. As far as the ambivalence of the self-dual shape is concerned, it is interesting to observe an oblate-to-prolate symmetry inversion in the $\mathrm{N}_{U}$ domain upon increasing density. Our modified version of the Onsager theory for monodisperse biaxial particles suggests that the free-energy difference between the $\mathrm{N}_{\mathrm{U}}^{-}$and $\mathrm{N}_{\mathrm{U}}^{+}$phases is very small, offering a possible explanation for the symmetry inversions observed in our simulations.

\section{Conflicts of interest}

There are no conflicts to declare.

\section{Acknowledgements}

EMR would like to thank the Malaysian Government Agency Majlis Amanah Rakyat for funding his $\mathrm{PhD}$ at the University of Manchester. AC acknowledges the Spanish Ministerio de Ciencia, Innovación y Universidades and FEDER for funding (project PGC2018-097151-B-I00) and C3UPO for the HPC facilities provided. AP acknowledges financial support from the EPSRC under grant agreement EP/N02690X/1 and the Leverhulme Trust Research Project Grant RPG-2018-415. We would like to thank Andrew Masters and Matthew Dennison for sharing the code to calculate the virial coefficients. Finally, EMR, DC and AP acknowledge the assistance given by IT Services and the use of the Computational Shared Facility at the University of Manchester.

\section{Notes and references}

1 L. Onsager, Ann. N. Y. Acad. Sci., 1949, 51, 627-659.

2 D. Frenkel, H. Lekkerkerker and A. Stroobants, Nature, 1988, 332, 822.

3 F. van der Kooij and H. Lekkerkerker, Phys. Rev. Lett., 2000, 84, 781.

4 B. R. Acharya, A. Primak and S. Kumar, Phys. Rev. Lett., 2004, 92, 145506.

5 L. A. Madsen, T. J. Dingemans, M. Nakata and E. T. Samulski, Phys. Rev. Lett., 2004, 92, 145505.

6 E. van den Pol, A. Petukhov, D. Thies-Weesie, D. Byelov and G. Vroege, Phys. Rev. Lett., 2009, 103, 258301.

7 M. Freiser, Phys. Rev. Lett., 1970, 24, 1041.

8 J.-H. Lee, T.-K. Lim, W.-T. Kim and J.-I. Jin, J. Appl. Phys., 2007, 101, 034105.

9 R. Berardi, L. Muccioli and C. Zannoni, J. Chem. Phys., 2008, 128, 024905.
10 M. Ricci, R. Berardi and C. Zannoni, J. Chem. Phys., 2015, 143, 084705.

11 M. P. Taylor and J. Herzfeld, Phys. Rev. A: At., Mol., Opt. Phys., 1991, 44, 3742.

12 Y. Martnez-Ratón, S. Varga and E. Velasco, Phys. Chem. Chem. Phys., 2011, 13, 13247-13254.

13 S. Belli, A. Patti, M. Dijkstra and R. van Roij, Phys. Rev. Lett., 2011, 107, 148303.

14 S. Belli, M. Dijkstra and R. van Roij, J. Phys.: Condens. Matter, 2012, 24, 284128.

15 S. D. Peroukidis and A. G. Vanakaras, Soft Matter, 2013, 9, 7419-7423.

16 S. D. Peroukidis, A. G. Vanakaras and D. J. Photinos, Phys. Rev. E: Stat., Nonlinear, Soft Matter Phys., 2013, 88, 062508.

17 S. D. Peroukidis, Soft Matter, 2014, 10, 4199-4207.

18 A. B. G. M. Leferink op Reinink, S. Belli, R. van Roij, M. Dijkstra, A. V. Petukhov and G. J. Vroege, Soft Matter, 2014, 10, 446-456.

19 Y. Martnez-Ratón, S. Varga and E. Velasco, J. Chem. Phys., 2014, 140, 204906.

20 L. Mederos, E. Velasco and Y. Martnez-Ratón, J. Phys.: Condens. Matter, 2014, 26, 463101.

21 M. González-Pinto, Y. Martnez-Ratón, E. Velasco and S. Varga, Phys. Chem. Chem. Phys., 2015, 17, 6389-6400.

22 A. Cuetos, M. Dennison, A. Masters and A. Patti, Soft Matter, 2017, 13, 4720-4732.

23 A. Patti and A. Cuetos, Mol. Simul., 2018, 44, 516-522.

24 S. Dussi, N. Tasios, T. Drwenski, R. van Roij and M. Dijkstra, Phys. Rev. Lett., 2018, 120, 177801.

25 Y. Yang, G. Chen, S. Thanneeru, J. He, K. Liu and Z. Nie, Nat. Commun., 2018, 9, 4513.

26 A. Cuetos, E. M. Rafael, D. Corbett and A. Patti, Soft Matter, 2019, 15, 1922-1926.

27 R. A. Skutnik, I. S. Geier and M. Schoen, Mol. Phys., 2020, $1-21$.

28 N. Tasios and M. Dijkstra, J. Chem. Phys., 2017, 146, 144901.

29 L. Querciagrossa, M. Ricci, R. Berardi and C. Zannoni, Phys. Chem. Chem. Phys., 2017, 19, 2383-2391.

30 S. Orlandi, L. Muccioli and R. Berardi, Liq. Cryst., 2018, 45, 2400-2415.

31 T. Drwenski and R. van Roij, Mol. Phys., 2018, 116, 2812-2822.

32 M. Chiappini, T. Drwenski, R. Van Roij and M. Dijkstra, Phys. Rev. Lett., 2019, 123, 068001.

33 A. Vanakaras, M. Bates and D. Photinos, Phys. Chem. Chem. Phys., 2003, 5, 3700-3706.

34 J. P. Straley, Phys. Rev. A: At., Mol., Opt. Phys., 1974, 10, 1881. 35 B. Mulder, Phys. Rev. A: At., Mol., Opt. Phys., 1989, 39, 360.

36 M. P. Allen, Liq. Cryst., 1990, 8, 499-511.

37 P. J. Camp and M. P. Allen, J. Chem. Phys., 1997, 106, 6681-6688.

38 M. A. Bates and D. Frenkel, J. Chem. Phys., 1998, 109, 6193-6199.

39 S. Gottschalk, M. C. Lin and D. Manocha, Comput. Graph, 1996, 30, 171-180. 
40 B. S. John, C. Juhlin and F. A. Escobedo, J. Chem. Phys, 2008, 128, 044909.

41 R. Alben, Phys. Rev. Lett., 1973, 30, 778.

42 E. van den Pol, D. Thies-Weesie, A. Petukhov, G. Vroege and K. Kvashnina, J. Chem. Phys., 2008, 129, 164715.

43 R. Berardi and C. Zannoni, J. Chem. Phys., 2000, 113, 5971-5979.
44 G. S. Preeti, K. Murthy, V. Sastry, C. Chiccoli, P. Pasini, R. Berardi and C. Zannoni, Soft Matter, 2011, 7, 11483-11487. 45 L. Querciagrossa, M. Ricci, R. Berardi and C. Zannoni, Phys. Chem. Chem. Phys., 2013, 15, 19065-19072.

46 L. Querciagrossa, R. Berardi and C. Zannoni, Soft Matter, 2018, 14, 2245-2253. 